\title{
Addendum to Ramachandra's paper "Some problems of analytic number theory, I"
}

\author{
by
}

\author{
K. Ramachandra, A. Sankaranarayanan and \\ K. SRinivas (Bombay)
}

To Professors

Yoichi Motohashi and Shigeru Kanemitsu

1. Introduction. The object of this note is to point out two immediate corollaries which can be obtained by the method of Ramachandra's paper [2] (mentioned in the title). Of course some explanation is necessary to point out what is new in this addendum. In the main theorem of Ramachandra's paper there are two epsilons (one in the definition of $\varphi$ and another in the definition of $\varphi^{\prime}$ ). These epsilons will be refined (as much as possible) as functions of $x$ and $X$ respectively. To do this we have to impose some restrictions on $b_{n}$ in $F_{0}(s)=\sum_{n=1}^{\infty} b_{n} n^{-s}$ which will be assumed to be absolutely convergent in $\operatorname{Re} s>1 / 2$. But this is not enough. We impose in addition the conditions (1) and (7) stated respectively in Corollaries 1 and 2 below. It should be mentioned that Corollary 2 is more involved in the sense that we need Theorem 2 of Section 2 below. We denote by $C_{1}, \ldots, C_{16}$ certain positive constants independent of $h, H, x$ and $X$.

Corollary 1. Let $\log L(s, \chi)$ be defined as in [2] for all L-series. Let $S_{4}$ denote a fixed finite set of these logarithms and their derivatives of bounded order. Let $P_{4}$ denote any power product (with bounded positive integers as exponents) of functions in $S_{4}$. Let $F_{0}(s)$ be as before but with the complex numbers $b_{n}$ subject to

$$
\left|b_{n}\right| \leq(\log (n+2))^{C_{1}} \quad\left(C_{1} \text { is a constant }\right) .
$$

Define $a_{n}$ by

$$
F(s)=P_{4}(s) F_{0}(s)=\sum_{n=1}^{\infty} a_{n} n^{-s} \quad(\operatorname{Re} s>1) .
$$


Let (with constants $B \geq 2$ and $D>0$ )

$$
N_{\chi}(\alpha, T)=O\left(T^{B(1-\alpha)}(\log T)^{D}\right) \quad(0 \leq \alpha \leq 1)
$$

in the usual notation (explained in [2]). Also let

$$
I(x, h)=\frac{1}{2 \pi i} \int_{0}^{h}\left(\int_{C_{0}} F(s)(v+x)^{s-1} d s\right) d v
$$

as in [2]. Put

$$
N(x)=\sum_{n \leq x} a_{n}, \quad \varphi=1-\frac{1}{B}+\frac{C_{2} \log \log x}{\log x}, \quad \varphi^{\prime}=1-\frac{2}{B}+\frac{C_{3} \log \log X}{\log X}
$$

for suitable constants $C_{2}$ and $C_{3}$. Then for $h=h(x), 1 \leq h \leq x$, we have

$$
N(x+h)-N(x)=I(x, h)+O\left(h \operatorname{Exp}\left(-(\log x)^{1 / 6}\right)+x^{\varphi}\right)
$$

and also for $h=h(X), 1 \leq h \leq X$, we have

(6) $\frac{1}{X} \int_{X}^{2 X}|N(x+h)-N(x)-I(x, h)|^{2} d x$

$$
=O\left(h^{2} \operatorname{Exp}\left(-(\log X)^{1 / 6}\right)+X^{2 \varphi^{\prime}}\right) .
$$

Remark. For reader's convenience we recall that $C_{0}$ is the contour $s=1+r e^{i \theta}(-\pi<\theta<\pi$ and $r$ is a sufficiently small positive constant).

Proof of Corollary 1. The proof is essentially the same as in [2]. We have to take $a=(\log T)^{-1}$. The Borel-Carathéodory theorem and Cauchy's theorem give the estimates $|F(s)| \leq(\log T)^{C_{4}}$ on $m(H H)$ in (23) of [2]. The only other changes are (i) to take $c=1+(\log x)^{-1}$ in (17) and consequently the $O$-term is $O\left(x(\log x)^{C_{5}} / T\right)$ for $2 \leq T \leq x$, and (ii) to select $T=x^{1 / B}(\log x)^{-C_{6}}$, where $C_{6}$ is a large constant. We are thus led to (5). To prove (6) we take $c=1+(\log X)^{-1}$ and set $T=X^{2 / B}(\log X)^{-C_{7}}$, where $C_{7}$ is a large constant.

COROLlary 2. Let $F_{0}(s)$ be as in [2], but now with the restriction

$$
\left|b_{n}\right| \leq \operatorname{Exp}\left(C_{8}(\log (n+2))(\log \log (n+20))^{-1}\right)
$$

where $C_{8}>0$ is a constant. Let $a_{n}(n=1,2, \ldots)$ be defined (as in [2]) by

$$
F(s)=P_{1}(s) P_{2}(s) P_{3}(s) F_{0}(s)=\sum_{n=1}^{\infty} a_{n} n^{-s} .
$$

Then subject to the condition (3) above and with $N(x), h, I(x, h)$ as described in Corollary 1, but with

$$
\varphi=1-\frac{1}{B}+\frac{C_{9}}{\log \log x}, \quad \varphi^{\prime}=1-\frac{2}{B}+\frac{C_{10}}{\log \log X},
$$


where $C_{9}$ and $C_{10}$ are suitable constants, we have

$$
N(x+h)-N(x)=I(x, h)+O\left(h \operatorname{Exp}\left(-(\log x)^{1 / 6}\right)+x^{\varphi}\right)
$$

and

$$
\begin{aligned}
\frac{1}{X} \int_{X}^{2 X}|N(x+h)-N(x)-I(x, h)|^{2} d x & \\
& =O\left(h^{2} \operatorname{Exp}\left(-(\log X)^{1 / 6}\right)+X^{2 \varphi^{\prime}}\right) .
\end{aligned}
$$

Remark. We briefly recall the notation for the convenience of the reader (for details see [2]). Let $S_{1}$ be the set of all Dirichlet $L$-functions, $S_{2}$ the set of all derivatives of all $L$-series in $S_{1}$ and $S_{3}$ the set of logarithms of all $L$-series in $S_{1} . P_{1}(s)$ is any finite power product (with complex exponents) of functions in $S_{1} . P_{2}(s)$ is any finite power product (with non-negative integral exponents) of functions in $S_{2} . P_{3}(s)$ is any finite power product (with non-negative integral exponents) of functions in $S_{3}$.

Proof of Corollary 2. The proof is essentially the same as in [2]. We have to take $a=(\log \log T)^{-1}$. The necessary estimate $|F(s)| \leq$ $\operatorname{Exp}\left(C_{11}(\log T)(\log \log T)^{-1}\right)$ in place of $T^{\varepsilon}$ in (23) of that paper is provided by some results of K. Ramachandra and A. Sankaranarayanan [4] which we state at the end of Section 2. The only other changes are (i) to take $c=1+$ $(\log x)^{-1}$ in (17) and consequently the $O$-term is $O\left(T^{-1} x \operatorname{Exp}\left(C_{12}(\log x) \times\right.\right.$ $\left.\left.(\log \log x)^{-1}\right)\right)$ for $2 \leq T \leq x\left(C_{12}>0\right.$ is some constant), and (ii) to select $T=x^{1 / B-C_{13} l}$, where $l=(\log \log x)^{-1}$ and $C_{13}$ is a large constant. These lead to (9). To prove (10) we take $c=1+(\log X)^{-1}$ and set $T=X^{2 / B-C_{14} l_{1}}$, where $l_{1}=(\log \log X)^{-1}$ and $C_{14}$ is a large constant. These lead to (10).

2. Some remarks. In (3) and hence in Corollaries 1 and 2 above we can take $B=12 / 5$ by a well-known result due to H. L. Montgomery and M. N. Huxley (see [2] for reference). The following special case of Corollary 2 is worth noting. We state it as a theorem.

Theorem 1. Let $1 \leq h \leq x, h=h(x)$ and $1 \leq H \leq X, H=H(X)$. Then there exist positive constants $C_{15}$ and $C_{16}$ such that

$$
\begin{aligned}
& \sum_{x \leq n \leq x+h} \mu(n) \\
& \quad=O\left(h \operatorname{Exp}\left(-(\log x)^{1 / 6}\right)+x^{7 / 12} \operatorname{Exp}\left(C_{15}(\log x)(\log \log x)^{-1}\right)\right)
\end{aligned}
$$

and

$$
\begin{aligned}
& \frac{1}{X} \int_{X}^{2 X}\left|\sum_{x \leq n \leq x+H} \mu(n)\right|^{2} d x \\
& =O\left(H^{2} \operatorname{Exp}\left(-(\log X)^{1 / 6}\right)+X^{1 / 3} \operatorname{Exp}\left(C_{16}(\log X)(\log \log X)^{-1}\right)\right) .
\end{aligned}
$$


R e mark. We can state similar results for the coefficients of $(\zeta(s))^{k}(k$ any non-zero complex constant; of course $C_{15}$ and $C_{16}$ will depend on $k$ ). But then $I(x, h)$ is negligible only when $k=-1,-2,-3, \ldots$

We next mention the theorem of K. Ramachandra and A. Sankaranarayanan (see [4]) used in the proof of Corollary 2. We nearly quote it from [1] where their result is stated in full generality. (One can also see [3] for some other uses of their result.) We adopt the notation of [1].

Theorem 2 (K. Ramachandra and A. Sankaranarayanan). Let

$$
D(s)=\sum_{n=1}^{\infty} a_{n} \lambda_{n}^{-s},
$$

where $a_{1}=\lambda_{1}=1,1 / A \leq \lambda_{n+1}-\lambda_{n} \leq A$ ( $A \geq 1$ is any constant), $\left\{\lambda_{n}\right\}$ is any sequence of real numbers and $\left\{a_{n}\right\}$ is any sequence of complex numbers with $\left|a_{n}\right| \leq n^{A}$. Let $\alpha>\delta(\delta(>0)$ a constant $)$ and let $R(H, \alpha)$ denote the rectangle $\left(\sigma \geq \alpha, T_{1}-H \leq t \leq T_{1}+H\right)$. Let $D(s)$ be continuable analytically in $R(H, \alpha-\delta)$ and there $\max |F(s)|<T^{A}$. Here $A_{5} \log \log \log T \leq H \leq T / 2$ and $T_{1}$ can be any number lying between $T$ and $2 T$. Let $D(s) \neq 0$ in $R(H, \alpha)$. Then for $t=T_{1}, s=\sigma+$ it in $R(H, \alpha)$ we have uniformly in $\sigma \geq \alpha, t=T_{1}$ the two inequalities

$$
\begin{array}{r}
-A_{1} \frac{\log T}{\log \log T} \max \left[1, \log \left(\frac{A_{2}}{(\sigma-\alpha) \log \log T}\right)\right] \\
\quad \leq \log |D(s)| \leq A_{3} \frac{\log T}{\log \log T}
\end{array}
$$

and

$$
|\arg D(s)| \leq A_{4} \frac{\log T}{\log \log T}
$$

Here $A_{1}, \ldots, A_{5}$ are positive constants depending only on $\delta$ and $A$.

Not e. It is enough to assume $D(s) \neq 0$ in $\left(\sigma>\alpha, T_{1}-H \leq t \leq T_{1}+H\right)$. Also the dependence of $A_{1}, \ldots, A_{5}$ on $\alpha$ is continuous in any closed bounded interval.

\section{References}

[1] R. Balasubramanian and K. Ramachandra, On Riemann zeta-function and allied questions, II, Hardy-Ramanujan J. 18 (1995), 10-22.

[2] K. Ramachandra, Some problems of analytic number theory, I, Acta Arith. 31 (1976), 313-324.

[3] - On the zeros of a class of generalised Dirichlet series, VII, Ann. Acad. Sci. Fenn. Ser. AI Math. 16 (1991), 391-397. 
[4] K. Ramachandra and A. Sankaranarayanan, On some theorems of Littlewood and Selberg, III, ibid., 139-149.

SCHOOL OF MATHEMATICS

TATA INSTITUTE OF FUNDAMENTAL RESEARCH

HOMI BHABHA ROAD, COLABA, BOMBAY 400 005, INDIA

E-mail: KRAM@TIFRVAX.TIFR.RES.IN

SANK@TIFRVAX.TIFR.RES.IN

SRINI@TIFRVAX.TIFR.RES.IN

Received on 20.9.1994

and in revised form on 20.4.1995 